\title{
Initiation but no execution - modulation of peripheral blood lymphocyte apoptosis in rheumatoid arthritis - a potential role for heat shock protein 70
}

\author{
Devapregasan Moodley ${ }^{1}$, Girish M Mody ${ }^{2}$ and Anil A Chuturgoon ${ }^{1 *}$
}

\begin{abstract}
Background: Rheumatoid arthritis (RA) is a chronic autoimmune disease, which causes synovial damage. Persistence of lymphocyte infiltrates in the rheumatoid synovium has been attributed to abnormal apoptosis. While not comprehensively investigated, perturbations in peripheral blood lymphocyte (PBL) apoptosis may also be involved in perpetuation of autoimmune processes in RA.

Methods: We investigated total, CD4+ and CD19+ PBL apoptosis in our study cohort by monitoring the translocation of phosphatidylserine using the Annexin- $V$ assay. To examine the role of death receptor mediated apoptosis as well as activation-induced-cell-death (AICD), PBLs were labeled with CD95/Fas and CD69 markers and enumerated by flow cytometry. Proteolytic activity of initiator and executioner caspases was determined by luminometry. DNA fragmentation assays were used to examine whether apoptotic signals were transduced to the nucleus. Quantitative PCR arrays were used to investigate apoptotic pathways associated with RA-PBLs. Since heatshock-protein-70 (HSP70) is an inducible protein which modulates apoptotic signals, we determined HSP70 levels by intra-cellular flow cytometry and western blots.
\end{abstract}

Results: The RA-PBLs showed signs of elevated apoptosis whilst in circulation. These include increases in the loss of plasma membrane asymmetry, indicated by increased externalization of phosphatidylserine (especially in Blymphocytes). RA-PBLs showed a bias to CD95/Fas mediated apoptotic pathways, but low levels of the CD69 marker suggested that this was not associated with immune activation. Although downstream markers of apoptosis such as caspase-3/7 activity, were increased, no DNA fragmentation was observed in RA-PBLs. Interestingly, elevated levels of apoptosis did not correlate with absolute lymphocyte counts in RA patients. Levels of HSP70 were highly elevated in RA-PBLs compared to controls.

Conclusion: The results suggest that while apoptosis may be initiated in RA-PBLs, they may lack commitment to fully executing the apoptotic program. This may be related to inhibition on apoptotic transduction by HSP70. This study provides evidence that abnormalities in RA-PBLs apoptosis may occur whilst still in circulation and may contribute to pathogenesis of the disease.

Keywords: Apoptosis, Lymphocyte, Rheumatoid arthritis, Heat shock protein

\footnotetext{
* Correspondence: chutur@ukzn.ac.za

'Discipline of Medical Biochemistry, Faculty of Health Sciences, University of

KwaZulu-Natal, Private Bag 7, Congella, 4013, Durban, South Africa

Full list of author information is available at the end of the article
} 


\section{Introduction}

Rheumatoid arthritis (RA) is an autoimmune disease characterized by chronic joint inflammation, synovial hyperplasia and bone erosion. In RA, the synovial microenvironment is considerably altered due to secretion of pro-inflammatory cytokines and subsequent infiltration of inflammatory cells. This facilitates pannus tissue formation and invasive growth of synovial tissue into articular cartilage and bone, with eventual joint destruction [1].

Bone and cartilage erosion occur during the natural progression of RA as a result of subtle underlying abnormalities in immune regulation and function. Accumulation and persistence of the lymphocyte infiltrate in the rheumatoid synovium are characteristic features of the disease [2]. In normal inflammatory responses, lymphocytes are eliminated, upon cessation of function, by initiation of apoptotic cascades [3]. Apoptosis is the major mechanism of programmed cell death and is necessary for regulation of tissue growth and homeostasis. In particular, the immune system relies heavily on apoptosis to ameliorate inflammation in order to prevent misdirected damage to normal tissue [4].

Several lines of evidence in RA suggest that malfunctions in apoptosis are responsible not only for the persistence of synovial lymphocytes, but also for the invasive nature of fibroblast-like-synoviocytes (FLS) [5,6]. Interactions between these cell types either through cellular contact or by secretion of soluble factors contribute to impaired apoptosis and chronic inflammation of the synovial membrane [7].

In murine models of proteoglycan induced arthritis, Tlymphocyte apoptosis was shown to be defective despite high expression levels of CD95/Fas and was related to impaired downstream CD95/Fas signaling pathways [8]. Elevated levels of anti-apoptotic Bcl-2 proteins conferred resistance to CD95/Fas-induced apoptosis in $\mathrm{CD}_{4}^{+}$ T-lymphocytes from RA patients [9]. Furthermore, it was shown that RA-FLS synthesize high quantities of stromal-cell-derived-factor-1 $\alpha$ (SDF1 $\alpha)$, a ligand for lymphocyte chemokine-receptor-4 (CXCR4), which induces migration of CD4 ${ }^{+} \mathrm{T}$-lymphocytes to the synovium. Interestingly, SDF1 $\alpha$ also inhibits T-lymphocyte apoptosis by interfering with mitogen-activated-protein-kinase (MAPK) pathways [10].

In addition to T-lymphocytes, there is growing interest in B-lymphocyte biology within the context of autoimmunity [11]. The recent success of anti-B-lymphocyte therapies support the notion that breakdown of normal B-lymphocyte function contributes to the pathogenesis of RA [12]. Indeed, there is accumulating evidence for impaired B-lymphocyte apoptosis in the rheumatoid synovium [13]. B-lymphocytes are enriched in the RA synovial membrane and are bound to FLS, which act as follicular dendritic cells [14]. In co-culture with RA synovial stromal cells, B-lymphocytes up-regulate expression of Bcl-xL, which inhibits mitochondrial pro-apoptotic signals [15]. Inhibition of B-lymphocyte apoptosis by FLS was shown to occur in a cell-contact dependant manner via vascular-cell-adhesion-molecule-1 (VCAM1) [16]. These data suggest that cell-contact interactions contribute to the pathophysiology ultimately leading to destruction of the rheumatoid synovium.

While synovial joints are the primary sites of inflammation in RA, there is a significant, but poorly understood systemic inflammatory component of the disease. Immuno-pathologies in RA are not limited to synovium sensitive cells but also involve the majority of circulating peripheral lymphocytes. Zhang et al (2001) proposed that defective apoptosis may lead to accumulation of T-lymphocytes in peripheral circulation [8]. It is likely that this may perpetuate the often elusive systemic complications of RA. There is compelling evidence to show that dysregulation of PBL apoptosis is critical in the pathogenesis of various systemic autoimmune diseases such as systemic lupus erythematosus (SLE), Sjögrens syndrome and systemic sclerosis $[17,18]$. Apoptosis in RA-PBLs however, has not been comprehensively investigated. Defects in RA-PBL apoptosis could clearly underlie some of the characteristic immunologic phenomena seen in RA patients.

Despite an unclear delineation of their specific roles, stress-response proteins, specifically heat shock proteins (HSPs), have been repeatedly implicated as key participators in the pathogenesis of RA [19]. Interestingly, numerous mechanisms of HSP-mediated inhibition of cell death have been described [20,21]. Since RA-PBLs exist in a milieu of inflammatory mediators such as tumor-necrosisfactor- $\alpha$ (TNF $\alpha$ ) and C-reactive protein (CRP) [22], it is likely that exacerbated stress responses may interfere with transmission of apoptotic signals.

It is generally accepted that the autoimmune manifestations of RA are due, in part, to impaired lymphocyte apoptosis. Whether these defects are related to failures in executing the apoptotic program at inflammatory sites, or as a result of inherent defects in lymphocyte apoptotic machinery prior to recruitment to these sites remain to be elucidated. The apoptotic status of circulating lymphocytes in RA may be a useful indicator of underlying pathological processes or disease activity. A better understanding of PBL biology, and indeed PBL apoptosis, may provide clues to how immunological tolerance is breached in RA. Consequently, in this study we investigated whether PBL apoptosis was impaired in RA patients directly ex vivo and whether there was an association with HSP70.

We report that PBL showed early signs of elevated apoptosis in our patient cohort and seemed to be associated with CD95/Fas, but is not necessarily related to 
lymphocyte activation. Interestingly, despite the significantly high levels of PBL apoptosis measured in patients, absolute lymphocyte counts remained high. In addition, we did not observe any downstream markers of apoptosis such as DNA fragmentation in patients. Our data also shows elevated levels of heat-shock-protein-70 (HSP70), which correlated with phosphatidylserine externalization. HSP70 is a known modulator of apoptosis and may interfere with transmission of apoptotic cascades in RA-PBLs. We suggest that abnormal control of PBL apoptosis may contribute to autoreactivity in persons who develop RA.

\section{Materials and methods}

Patient recruitment

Fifty South African black RA patients attending the Rheumatology clinic at Inkosi Albert Luthuli Central Hospital (Durban, South Africa) were recruited into the study. All patients (mean age: 50.7 years, range 18-75 years; mean duration of disease: $13.3 \pm 9.5$ years) fulfilled the American College of Rheumatology (ACR) criteria for RA [23] and were on disease-modifying antirheumatic drug (DMARD) treatments. Patient recruitment commenced following institutional ethical approval (H109/04) and informed consent was obtained for each patient. The patients reported no recent/ chronic infection or history of other chronic inflammatory diseases. Clinical and laboratory parameters (mean number of swollen joints and tender joints; erythrocyte sedimentation rate, ESR; C-reactive protein, CRP; absolute lymphocyte counts) were recorded for all the patients, (Table 1). An equal number of healthy race matched control samples were sourced from the South African National Blood Services following routine screening. Both control and patient groups had a female to male ratio of $6: 1$. Control samples were extracted and assayed in the same manner as patient samples to ensure comparability.

Table 1 Summary of clinical and laboratory parameters measured in RA patients

\begin{tabular}{lc}
\hline Parameter & Result (Mean \pm SD) \\
\hline Mean number of swollen joints & $12 \pm 6.7$ \\
Mean number of tender joints & $13 \pm 8.7$ \\
ESR & $42.3 \pm 28.5 \mathrm{~mm} / \mathrm{hr}$ \\
CRP & $19.59 \pm 20.5 \mathrm{mg} / \mathrm{ml}$ \\
Mean lymphocyte count & 13 \\
\hline Patients Treatments: & 29 \\
Patients on MTX & 8 \\
Patients on MTX + steroids & \\
Patients on other DMARDs & 22 \\
\hline Disease Duration: & 28 \\
$<10$ years & \\
$>10$ years & \\
\hline
\end{tabular}

\section{Peripheral blood mononuclear cell (PBL) preparation}

Buffy coats containing PBL were extracted from heparinized whole blood by differential centrifugation. Briefly, $5 \mathrm{ml}$ whole blood collected from each subject, was layered onto equivolume Histopaque 1077 (Sigma, Germany) in $15 \mathrm{ml}$ polypropylene tubes. Layered blood was then centrifuged at $400 \times \mathrm{g}$ for 30 minutes. Buffy coats were aspirated into new polypropylene tubes and washed twice in phosphate buffered saline $(\mathrm{PBS})(400 \times \mathrm{g}, 10$ minutes). Cell density was adjusted to $1 \times 10^{6}$ cells $/ \mathrm{ml}$ after exclusion of dead cells with trypan blue. Sample preparation and subsequent apoptosis assays were complete within 3-4hrs after drawing of blood from study participants.

\section{Detection of phosphatidylserine on outer membrane of peripheral lymphocytes}

The annexin-V-FITC apoptosis detection kit (Roche, Germany) was used to label apoptotic PBL with translocated phosphatidylserine residues on the outer plasma membrane. In addition to annexin-V-FITC, the kit contained propidium iodide (PI) which was used to monitor late stage apoptosis and necrotic cell death. The assay procedure was as per manufacturer's instructions. Briefly, annexin-V-FITC labeling solution $(100 \mu \mathrm{l})$ was added to $1 \times 10^{6} \mathrm{PBL}$ in cytometry tubes and allowed to incubate for 15 minutes in the dark at room temperature (RT). Following incubation, two separate aliquots of the annexin-labeled PBL were prepared in order to assess apoptosis in B- and T-lymphocyte sub-populations. Allophycocyanin (APC)-labeled anti-CD4 and anti-CD19 (Pharmingen, USA) was added $(5 \mu \mathrm{l})$ to the respective PBL aliquots 10 minutes prior to enumeration by flow cytometry.

\section{Detection of CD95/Fas and activation marker CD69 on peripheral lymphocytes}

Aliquots of approximately $1 \times 10^{5} \mathrm{PBL}$ were transferred into cytometry tubes containing monoclonal mouse antiCD95/Fas (1:100) (Sigma, Germany). The mixture was allowed to react for 20 minutes and PBL were thereafter washed in PBS $(400 \times$ g, 10 minutes). To detect Fas-positive PBL by flow cytometry, rabbit anti-mouse APClabeled secondary antibody (Sigma, Germany) was added to cells at a final dilution of 1:1000 and allowed to react for 15 minutes. In order to determine the activation status of circulating lymphocytes in RA patients, $1 \times 10^{5} \mathrm{PBL}$ were incubated with $10 \mu \mathrm{l}$ fluorescein-isothiocyanate (FITC) labeled anti-CD69 (BD Biosciences) for 15 minutes prior to analysis by flow cytometry.

Intra-cellular detection of heat shock protein 70 (HSP70) For each sample, $1 \times 10^{5} \mathrm{PBL}$ were transferred into cytometry tubes. Cells were then fixed $(100 \mu \mathrm{l}$ Caltag reagent 
A fixative medium; Caltag Laboratories, USA) for 15 minutes at RT. After fixation, PBL were washed in PBS supplemented with $0.1 \%$ sodium azide and $5 \%$ fetal bovine serum $(300 \times \mathrm{g}, 5$ minutes). Thereafter, $\mathrm{PBL}$ were re-suspended in permeabilization medium $(100 \mu \mathrm{l}$, Caltag reagent $\mathrm{B}$ permeabilization medium; Caltag Laboratories, USA) containing monoclonal mouse anti-HSP70 (1:1, 000; Pharmingen, USA) for 30 minutes. Following permeabilization and incubation with primary antibody, PBL were washed twice as previously described. Samples were then incubated with APC-conjugated rabbit anti-mouse secondary antibody (1:10, 000; Pharmingen, USA) for 20 minutes at RT in the dark. After an additional wash step, labeled PBL were re-suspended in sheath fluid for detection by flow cytometry.

\section{Flow cytometry}

PBL were identified and sorted according to forward and side angle scatter morphological parameters (Figure 1A). Labeled PBL in the assays above were enumerated by flow cytometry using a 4-colour FACS Calibur (BD Biosciences, Belgium) flow cytometer. Data was acquired with CellQuest Pro software (BD Biosciences, Belgium) from 100, 000 events for each assay. Analysis was performed with FlowJo 7.1 software (Tree Star Inc., USA).

\section{Apoptotic protease activity}

Luminometry assays determined the activities of apoptotic initiator caspase 9 and executioner caspases 3/7. Separate aliquots of PBL $\left(1 \times 10^{5}\right)$ were transferred into luminometry-quality white microtitre plates. $100 \mu \mathrm{l}$ of caspase substrate (Caspase-Glo 3/7, Caspase-Glo 9; Promega, USA) was added to PBL and allowed to react for 30 minutes. Luminescent signals were then measured with the Modulus microplate luminometer (Turner Biosystems, USA) and expressed as relative light units.

\section{Analysis of apoptotic pathways by quantitative PCR (qPCR) array}

RNA was extracted and purified from PBLs using Trizol reagent (Invitrogen, USA) as per manufacturer's instructions and thereafter quantified by spectrophotometry. Expression of 84 critical genes which represent 10 functional gene groups involved in apoptotic signal transduction pathways were analyzed by quantitative PCR using the Human Apoptosis $\mathrm{RT}^{2}$ Profiler PCR array (SABiosciences, USA), according to the manufacturer's protocol. Details regarding the technology and specific genes can be found at the following URL: http://www.sabiosciences.com/rt_pcr_product/HTML/PAHS-012A.html.

Briefly, cDNA was prepared from $1 \mu \mathrm{g}$ of total RNA using the $\mathrm{RT}^{2} \mathrm{PCR}$ array first strand kit (SABiosciences, USA). qPCR reactions were conducted in a $25 \mu \mathrm{lmix}$ ture which included $12.5 \mu \mathrm{l}$ of $2 \mathrm{X}$ qPCR master mix, $11.5 \mu \mathrm{l}$ of nuclease free water and $1 \mu \mathrm{l}$ of cDNA template. The thermal cycle profile consisted of an initial 10 minute step at $95^{\circ} \mathrm{C}$ followed by 40 cycles of $95^{\circ} \mathrm{C}$ for 15 seconds and $60^{\circ} \mathrm{C}$ for 1 minute. Real time quantitations were carried out using the Bio-Rad Chromo4 real time detection system (Bio-Rad, USA). Data was analyzed using SABiosciences web-based qPCR data analysis service. Fold changes were calculated using the $2^{-\Delta \Delta \mathrm{Ct}}$ method.

\section{Detection of HSP70 by Western blot}

Total PBL protein was extracted from each sample using Cytobuster $^{\mathrm{TM}}$ (Calbiochem, UK) reagent, supplemented with protease inhibitors, as per manufacturer guidelines. Protein concentration was determined by the bicinchoninic acid assay (Sigma, Germany) and standardized to $250 \mu \mathrm{g} / \mathrm{ml}$. Samples containing $10 \mu \mathrm{g}$ of protein were boiled in Laemmli buffer for 5 minutes and then subjected to electrophoresis in $10 \%$ sodium dodecyl sulfate
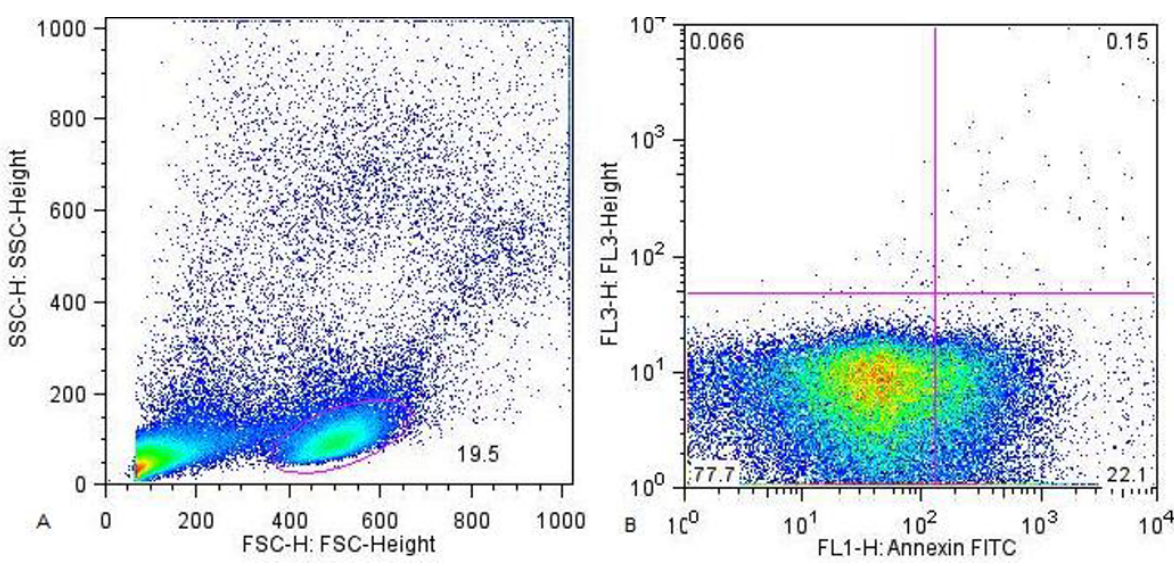

Figure 1 Flow cytometry scatter plots for the Annexin-V assay. A: PBL were identified and gated based on forward and side scatter properties. B: Scatter plots for green and red fluorescence channels were used to measure Annexin- $V$ and PI positivity respectively. 
(SDS)-polyacrylamide gels. Separated proteins were then electro-transfered to polyvinylidene difluoride membranes (PVDF). After blocking with Tris-buffered saline (TBS) containing 5\% nonfat dry milk and $0.1 \%$ Tween 20 , the membrane was immuno-probed with monoclonal anti-HSP70 (1:5, 000; Pharmingen, USA) for 1 hour at RT. The PVDF membrane was then subjected to 5 washes (10 minutes each) with TBS containing $0.1 \%$ Tween 20. The membrane was then exposed to secondary antibody (anti-mouse-horse-radish-peroxidase (HRP)-conjugate; 1:10, 000; Bio-Rad, USA) for 1 hour at RT. Anti- $\beta$-actin-HRP (Sigma, Germany) was utilized for internal loading controls. After further washing, antigen-antibody complexes were detected by chemiluminescence using the Immune-star ${ }^{\mathrm{TM}}$ HRP substrate kit (Bio-Rad, USA). Chemiluminescent signals were detected with the Chemi-doc XRS gel documentation system. Images were acquired and analyzed with Quantity-one $^{\mathrm{TM}}$ image analysis software (Bio-Rad, USA). Data is represented as peak band intensity for each sample.

\section{DNA fragmentation assay}

Genomic DNA was extracted from PBL $\left(1 \times 10^{5}\right)$ for each sample. Cells were transferred to $500 \mu \mathrm{l}$ lysis buffer containing $0.5 \%$ SDS, $150 \mathrm{mM} \mathrm{NaCl}, 10 \mathrm{mM}$ EDTA, and $10 \mathrm{mM}$ Tris- $\mathrm{HCl}$ (pH 8.0). To this RNase A $(100 \mu \mathrm{g} / \mathrm{ml}$; DNase-free) was added and the solution was incubated at $37^{\circ} \mathrm{C}$ for 1 hour. Subsequently proteinase K $(200 \mu \mathrm{g} / \mathrm{ml})$ was added to the solution and incubated for a further 3 hours at $50^{\circ} \mathrm{C}$. Protein contaminants were then precipitated by addition of 0.1 volume $5 \mathrm{mM}$ potassium acetate and centrifugation (5,000 $\times$ g; 15 minutes). Supernatants containing genomic DNA were transferred to fresh tubes and extracted with 100\% isopropanol on ice, and thereafter washed with $70 \%$ ethanol. DNA samples were then dissolved in $10 \mathrm{mM}$ Tris and $0.1 \mathrm{mM}$ EDTA (pH 7.4) at $4^{\circ} \mathrm{C}$ overnight. Concentration of each sample was determined spectrophotometrically. To prepare a positive control for the DNA fragmentation assay, apoptosis was induced in control PBL samples by treating with camptothecin $(4 \mu \mathrm{g} /$ $\mathrm{ml}, 12$ hours) in vitro. DNA was extracted and quantified as described above. Equal amounts of DNA (300ng) were electrophoresed (150V; 50 minutes) on a 1.8\% agarose gel containing $0.5 \mathrm{mg} / \mathrm{ml}$ ethidium bromide. DNA bands were visualized by UV light and digitally photographed using a gel documentation system and Quantity-one ${ }^{\mathrm{TM}}$ image analysis software (Bio-Rad, USA). Quantity-one ${ }^{\mathrm{TM}}$ software was used to quantify the density of bands for comparison between experimental groups.

\section{Statistical analysis}

Non-parametric Mann-Whitney tests were used to test for statistical differences between patients and controls for all apoptosis assays. Pearson, or Spearman rank correlations were used to test the dependence of variables where appropriate. All analyses were performed using the GraphPad Prism (V5) software package (GraphPad Software Inc., USA).

\section{Results}

\section{Elevated phosphatidylserine externalization in RA-PBL}

Translocation of phosphatidylserine residues from the inner leaflet of the plasma membrane to the outer leaflet of the plasma membrane is an early apoptotic event. Annexin- $\mathrm{V}$ is a specific and strong phosphatidylserinebinding protein [24] that detects cells undergoing apoptosis. The annexin- $\mathrm{V}$ assay showed that apoptosis was significantly higher $(\mathrm{p}<0.05)$ in RA PBL than in healthy controls ex vivo. When analyzed separately, apoptosis in RA CD4 ${ }^{+}$PBL was approximately 3.5 -fold higher than controls $(26.3 \pm 1.6 \%$ vs. $7.6 \pm 0.8 \%)$. The highest apoptosis values were recorded in the RA CD $19^{+} \mathrm{PBL}$, which were approximately 4-fold higher than controls (60.5 \pm $7.4 \%$ vs. $16.1 \pm 1.9 \%$; Table 2 ).

Late stage apoptotic and/or necrotic cells which take up PI were distinguished from PBL which were exclusively positive for annexin-V (Figure 1). The percent of RA-PBL positive for PI was extremely low $(0.4 \% \pm 0.10)$ and did not differ significantly from controls $(0.2 \pm 0.03$; $\mathrm{p}=0.1085$, unpaired $\mathrm{t}$ test).

To examine the effect of elevated apoptosis on the number of circulating lymphocytes in RA patients, we statistically tested whether total PBL apoptosis correlated with absolute lymphocyte counts. We found no statistically significant relationship between total PBL apoptosis and absolute lymphocyte counts (Figure 2).

In order to determine whether disease duration affected apoptosis levels in our study cohort, we examined whether apoptosis correlated with disease duration. We found that levels of apoptosis did not correlate with disease duration (Spearman rank correlation, $r=-0.1539$, $\mathrm{p}=0.2962)$. Patients were then grouped, i.e. patients with RA for $<10$ years $(n=22)$ and $>10$ years $(n=28)$. Although total PBL apoptosis was slightly higher in patients with RA for less than 10 years (30.6\% vs. $27.8 \%$ ), disease duration did not significantly affect apoptosis in

\section{Table 2 Annexin-V analysis of apoptosis in peripheral lymphocyte sub-populations from South African rheumatoid arthritis patients and race-matched controls ex vivo}

\begin{tabular}{lcc}
\hline $\begin{array}{l}\text { Cell } \\
\text { population }\end{array}$ & Patients [Mean \% (SEM)] & Controls [Mean \% (SEM)] \\
\hline Total PBL & $30.0(1.5)^{*}$ & $7.2(0.9)$ \\
CD4 ${ }^{+}$PBL & $26.3(1.6)^{*}$ & $7.6(0.8)$ \\
CD19 ${ }^{+}$PBL & $60.5(7.4)^{*}$ & $16.1(1.9)$ \\
\hline
\end{tabular}

* Significant difference ( $p<0.05$; Mann-Whitney test). CD4, cytotoxic

T-lymphocyte marker; CD19, pan B-lymphocyte marker. 


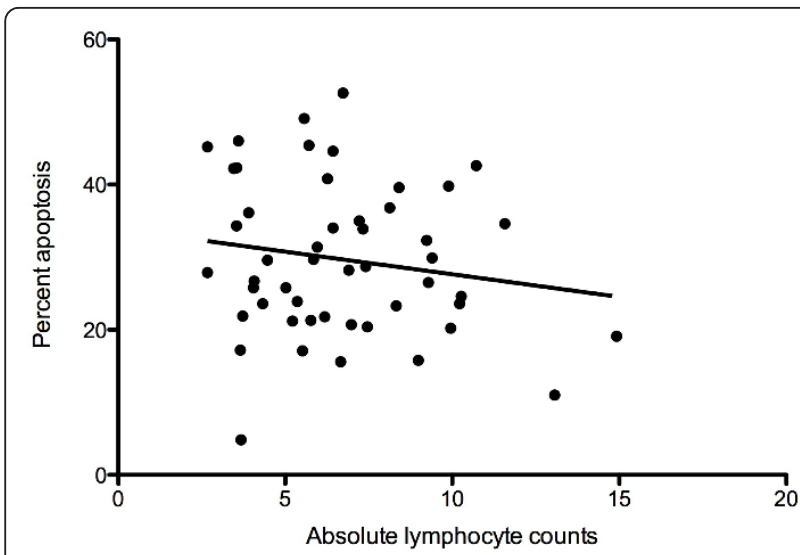

Figure 2 Absolute lymphocyte counts did not correlate with peripheral lymphocyte apoptosis in RA patients. Correlation coefficient $r=-0.08956$; $p$ value $=0.5362$; Pearson $r$ correlation.

our patient cohort ( $p=0.6843$; unpaired t test with Welch correction).

Patients were at various stages of treatment when sampled. They were distributed among three treatment categories, namely, patients on methotrexate (MTX) alone $(\mathrm{n}=13)$, MTX together with steroidal drugs $(\mathrm{n}=29)$ and those on other disease-modifying-anti-rheumatic-drugs (DMARD, $\mathrm{n}=8$ ). There was no significant difference in PBL apoptosis ( $p=0.6967$, one way ANOVA; Figure 3 ) when compared across different treatments. Experimental values measured in our patient cohort were independent of treatment.

\section{Higher percentage of RA-PBL with CD95/Fas on plasma} membrane

To determine whether the elevated apoptosis measured in our patient cohort was associated with receptor mediated apoptosis-inducing signals, we tested for the presence of CD95/Fas on PBL. The proportion of PBL

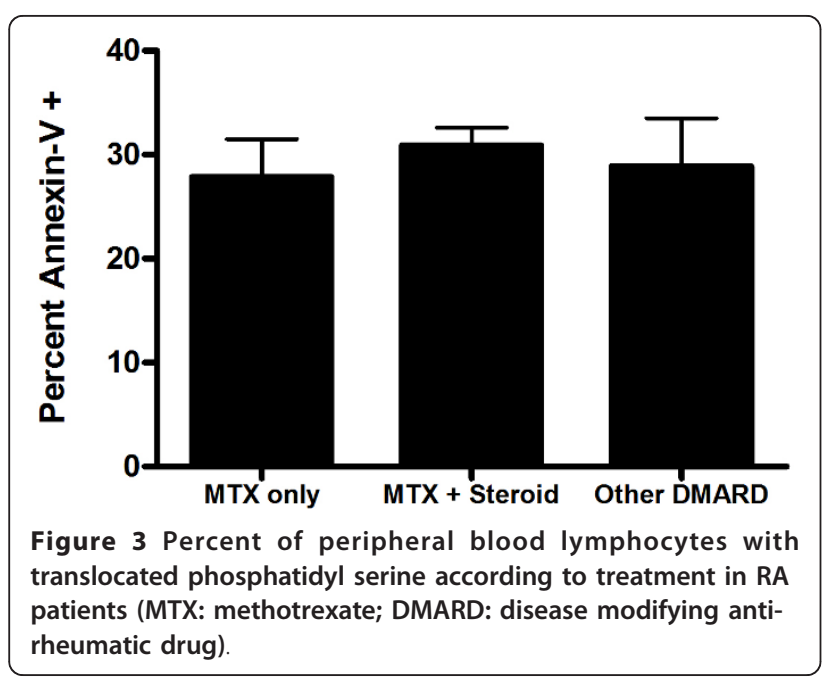

expressing CD95/Fas was significantly higher in RA patients compared to controls $(p=0.0317$; Mann Whitney test; Figure 4).

\section{Increased expression of Fas pathway associated transcripts in RA-PBLs}

To investigate apoptotic signaling pathways in RA-PBLs, we analyzed the expression profiles of 84 genes involved in the induction and/or transduction of apoptosis. The highest fold change differences were observed in genes associated with the Fas-mediated apoptotic pathway (Figure 5).

\section{RA-PBL showed low levels of activation}

Since CD95/Fas is associated with AICD in lymphocytes, we examined the activation status of circulating lymphocytes in our study cohort by monitoring the proportion of PBL positive for the CD69 activation marker. Interestingly, despite high apoptosis levels, RA patients had a lower percent of PBL positive for CD69 compared to controls, however, this did not reach statistical significance ( $p>0.05$; Mann Whitney test; Figure 6).

\section{Elevated caspase activity in RA-PBL}

Despite low luminescent signals recorded for executioner caspase $3 / 7$ activity, there was approximately 3 -fold higher activity in RA PBL compared to healthy controls. This difference in activity reached statistical significance $(p<0.01$; Mann-Whitney test; Figure 7A). Caspase-9 activity produced strong luminescent signals in both patients and controls, but was not statistically significant between these groups (Figure 7B).

\section{Elevated HSP70 in RA-PBL}

Since HSP70 is an inducible protein which can modulate apoptosis signals, the levels of HSP70 in PBL were

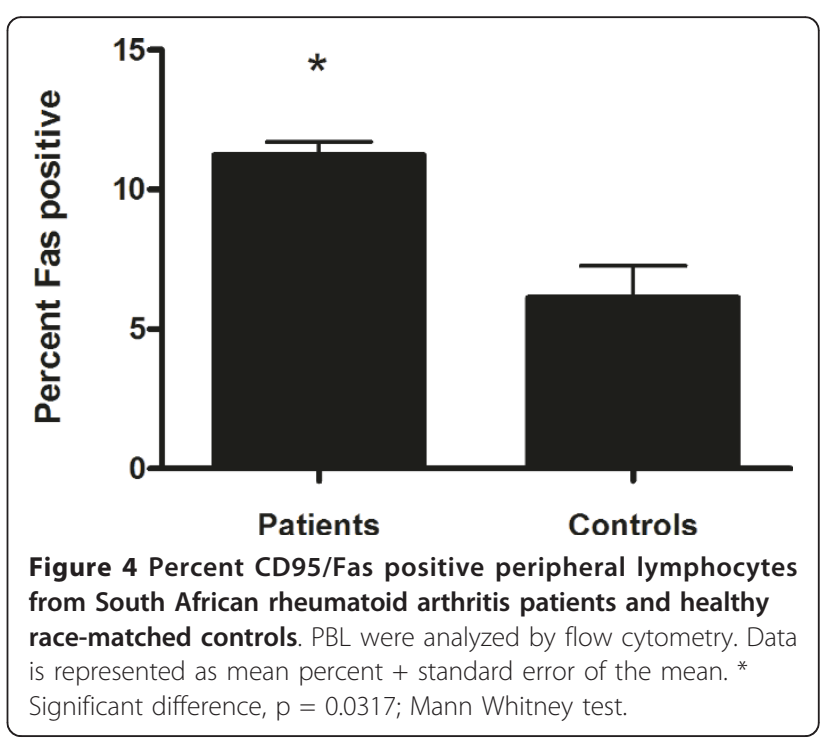




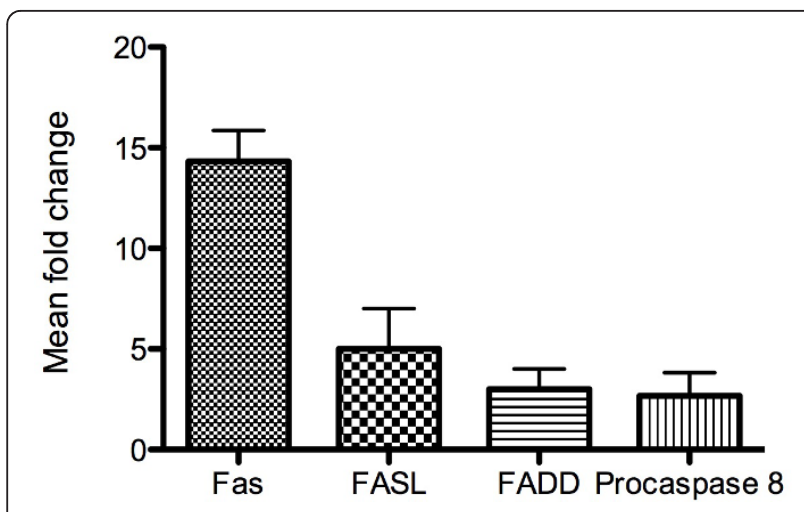

Figure $\mathbf{5}$ Increased expression of Fas pathway associated genes in RA-PBL. Data is represented as mean fold change + standard error of the mean.

examined. Using intra-cellular flow cytometry, we distinguished between PBL with high or low levels of intra-cellular HSP70 as a function of mean fluorescence intensity (Figure 8A). Our data showed that the proportion of PBL with detectable levels of intra-cellular HSP70 was significantly higher in RA patients compared to controls ( $p=$ 0.0001; Mann-Whitney test; Figure 8B). To confirm these data, western blot analysis for HSP70 was performed on total PBL protein (Figure $8 \mathrm{C}$ ). Band analyses showed that HSP70 levels were significantly elevated in RA patients ( $p$ $=0.0090$; unpaired t-test; Figure 8D). We next investigated whether there was an association between HSP70 levels and phosphatidylserine externalization in RA-PBLs. Our data showed a strong positive correlation between HSP70 levels and phosphatdylserine externalization in RA-PBLs (Figure 9).

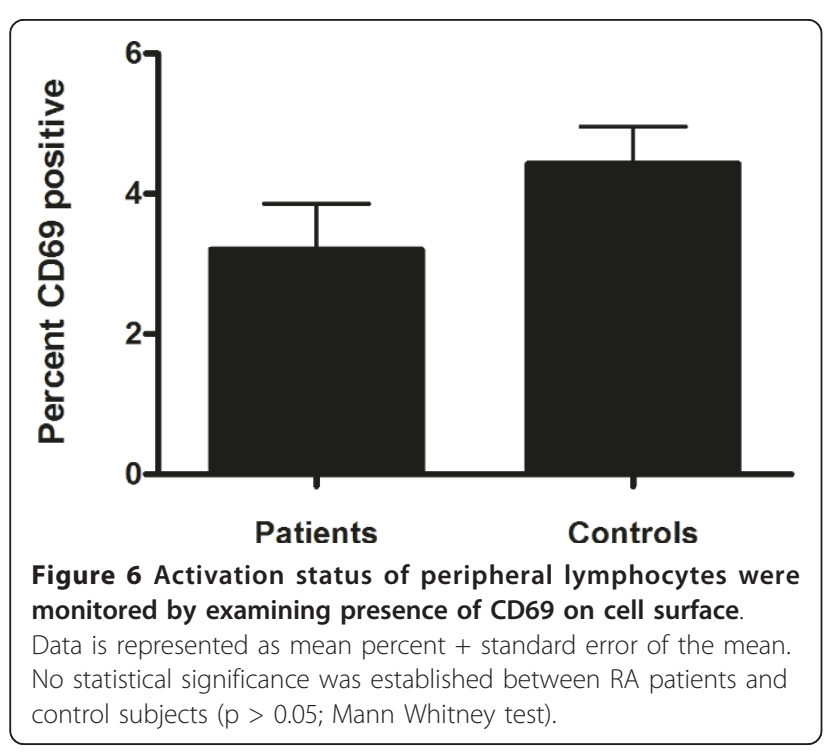

\section{No DNA fragmentation in RA-PBL}

DNA fragmentation, a typical molecular feature of apoptosis, occurs due to nuclease mediated cleavage of genomic DNA into oligonucleosomal fragments in multiples of approximately 200 base pairs. Nuclease activity is induced towards the latter stages of apoptosis by upstream apoptosis mediators such as caspase-3. The characteristic apoptosis DNA fragmentation pattern was not observed in patient or control samples following agarose gel electrophoresis. All DNA bands were of high molecular weight and intact with no signs of apoptosis-induced DNA damage (Figure 10).

\section{Discussion}

The fine balance between cell survival and cell death is essential for homeostasis in multi-cellular organisms. Apoptosis is the major mechanism of physiological cell death which facilitates deletion of unwanted or damaged cells. It plays a central role in the immune system in both the maintenance of self-tolerance and homeostatic control of lymphocyte populations [4]. The immune system relies on apoptosis for its functional integrity at multiple levels, and consequently, stringent regulation of these pathways is imperative. Immunological tolerance is promoted by carefully directed apoptosis in self-reactive $\mathrm{T}$-lymphocyte clones during their maturation in the thymus [25]. Immune learning continues while lymphocytes are in peripheral circulation, since not all antigenic combinations are encountered in the thymus. The precise mechanisms of peripheral immune learning are unknown, but may also involve deletion of self-reactive lymphocytes by apoptosis whilst in circulation [26]. It is likely that defects in peripheral immune learning may lead to autoimmunity. Lymphocyte death is tightly regulated and there are detrimental consequences when regulatory mechanisms are compromised. For instance, abnormal increases in apoptosis can cause immunodeficiency [27]; while a failure to undergo apoptosis can lead to development of autoimmunity [28].

In RA, there is compelling evidence to show that a compromise in lymphocyte apoptosis contributes to the persistence of these cells at inflamed joints [6]. Inflammation is normally resolved by carefully directed apoptosis of invading immune cells [4]. In RA however, the molecular interactions between synovial cells and infiltrating lymphocytes have been shown to protect against apoptosis in the synovium [8].

Little is known about the biological status of circulating lymphocytes prior to synovial recruitment in RA. Our studies therefore focused on circulating lymphocytes, which perpetuate the autoimmune manifestations of RA. We assessed PBL apoptosis since they are exposed to a myriad of pro-inflammatory cytokines and acute-phase proteins. This may compromise functional integrity 

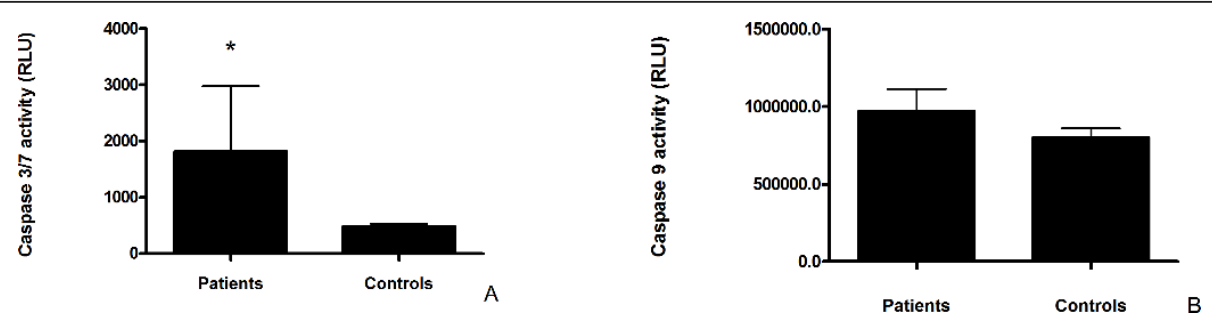

Figure 7 Apoptotic protease activity in peripheral lymphocytes. A: Higher caspase 3/7 activity in RA-PBL despite low luminescent signals. B Initiator caspase 9 activity was high in both study groups but not significantly different. Data is expressed as mean relative light units + standard error of the mean. * Significant difference, $p<0.01$; Mann-Whitney test.

before the PBL adopt a stationary phenotype in the rheumatoid synovium. Our data showed that early markers of apoptosis were elevated in total lymphocytes from RA patients. This trend was mimicked in $\mathrm{CD} 4^{+}$lymphocytes, but more so in $\mathrm{CD} 19^{+}$B-lymphocytes; where more than half of this lymphocyte population showed apoptotic features whilst in circulation. This suggests that abnormalities in the regulation of lymphocyte apoptosis may occur early in RA, prior to synovial infiltration.

Our data also showed minor elevations in control PBL apoptosis compared to baseline values previously reported in the literature. This may be indicative of normal baseline

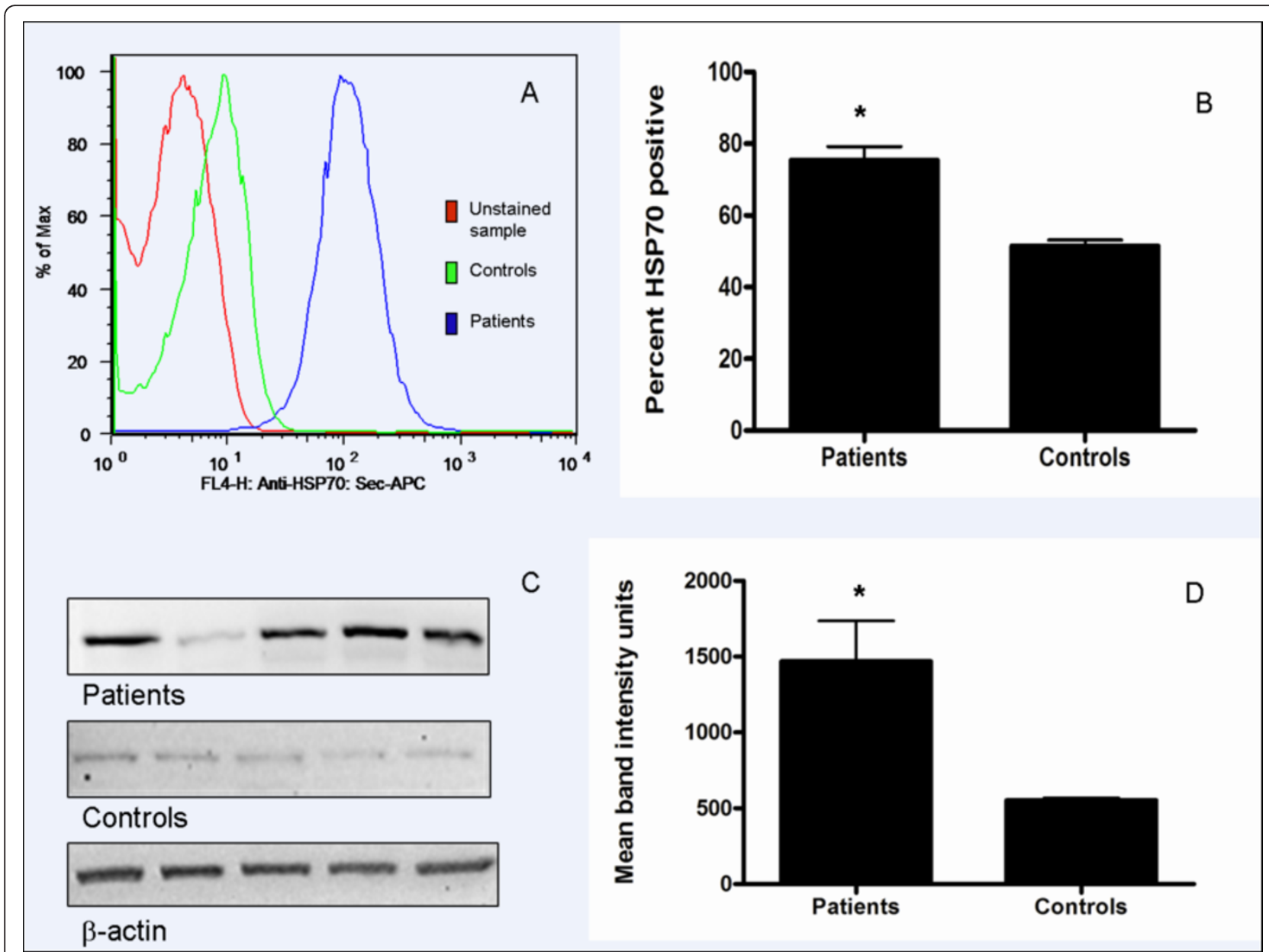

Figure 8 Detection of HSP70 in peripheral lymphocytes. A: Mean fluorescence intensity histogram for intra-cellular detection of HSP70 by flow cytometry. B: Proportion of PBL with detectable levels of HSP70 was higher in RA patients (* $p=0.0001$; Mann-Whitney test). C: Representative western blot analysis of HSP70 in total PBL protein. D: HSP70 levels significantly elevated in RA patients ( ${ }^{*} p=0.0090$; unpaired ttest). 


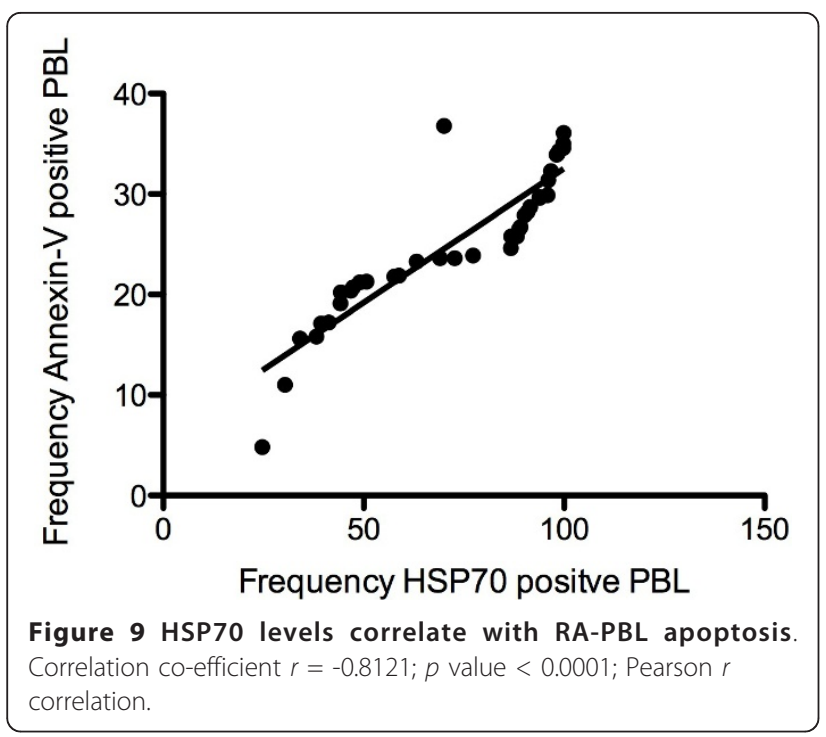

fluctuations in apoptosis levels since lymphocytes are extremely sensitive to physiological changes. Considering baseline apoptotic noise, it is likely that observations of relative apoptotic changes, which deviate significantly in disease phenotypes, may in fact indicate an integral role for the apoptosis in disease progression or etiology.

The elevated levels of PBL apoptosis observed in our patient cohort was not associated with AICD, since only a small percentage of lymphocytes showed detectable levels of the CD69 activation marker. CD69 is a transiently expressed membrane receptor early during lymphocyte activation, but is also selectively expressed in chronic inflammation [29]. Interestingly, engagement of the CD69

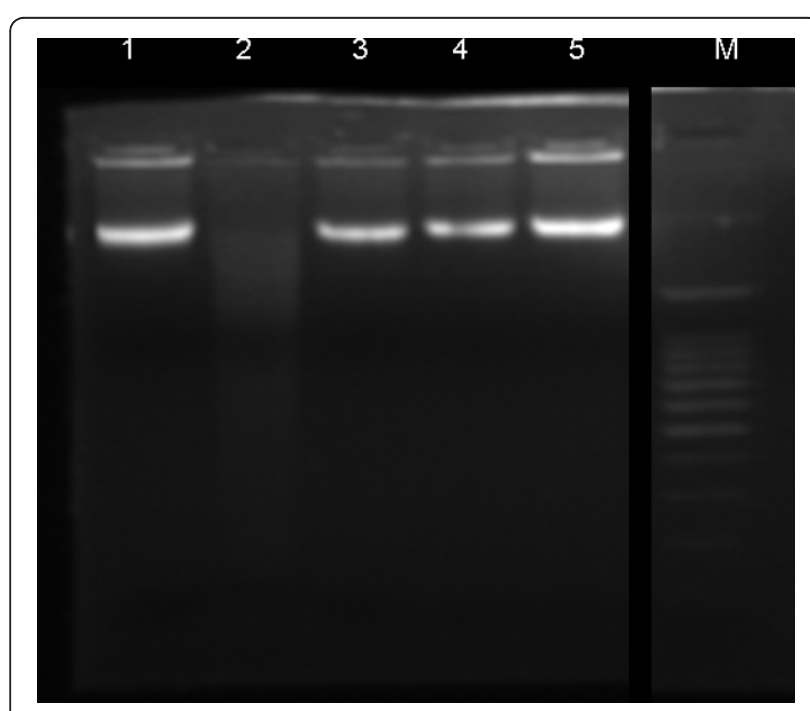

Figure 10 DNA fragmentation assay in peripheral lymphocytes by agarose gel electrophoresis. M: molecular weight marker; lane 1: control PBL DNA; lane 2: positive control; lane 3, 4, 5: RA-PBL DNA. receptor was shown to trigger apoptosis in multiple cell types [30], but despite persistent expression in chronic inflammatory infiltrates, lymphocyte apoptosis was inhibited. Evidence from molecular and cellular studies showed that T-lymphocyte activation was altered in RA [31]. This may account for the high levels of RA-PBL apoptosis observed in our patients where signs of early lymphocyte activation were relatively absent.

Under normal conditions, healthy mitochondria have polarized electronegative transmembrane gradients due to oxidative phosphorylation reactions. We have previously reported that mitochondrial depolarization was elevated in RA-PBL [32]. Loss of transmembrane potential alters mitochondrial permeability which results in the release of proteins such as cytochrome $c$ and second-mitochondrial activator of caspases/direct-inhibitor-of-apoptosis-bindingprotein-with-low-PI (Smac/DIABLO) into the cytoplasm [33]. In the cytoplasm, cytochrome $c$ binds to apoptoticprotease-activating-factor (Apaf-1) and pro-caspases, leading to ATP-dependant formation of the apoptosome [34]. The apoptosome is a potent activator of initiator caspases, in particular caspase-9. Activated caspase-9 in turn facilitates activation of executioner caspases (primarily caspase3 and caspase-7), which co-ordinate proteolytic breakdown of apoptotic cells. Engagement of CD95/Fas with its ligand leads to activation of caspase- 8 following recruitment of Fas-associated-death-domain-protein (FADD). This can signal apoptosis via two well-described pathways: (i) direct activation of caspase-3; or (ii) alteration of mitochondrial transmembrane potential via $\mathrm{Bcl}-2$ homology-3 (BH3)-interacting-death-domain (BID) agonist, which initiates the mitochondrial apoptosis cascade $[35,36]$. The CD95/Fas signaling pathway ultimately culminates in the activation of executioner caspases, which is a molecular hallmark of apoptosis.

Although caspase-3/7 activity was significantly higher in RA patients, these activities remained relatively low. This suggests that there may be perturbations in the signaling pathways which activate executioner caspases in these cells. A possible mechanism may involve HSP mediated interference between apoptosis initiator signals and their down-stream targets.. Activation of caspase-3 for instance, is suppressed by HSP27 since it binds to pro-caspase-3, thus preventing its activation by caspase- 9 [37]. Alternatively, HSP27 may sequester cytochrome $c$ from Apaf-1, thus preventing assembly of the apoptosome $[38,39]$. In addition the small HSP $\alpha \beta$ crystalline, suppresses cytochrome $c$-mediated autoactivation of caspase-3, by direct interaction with the enzyme to prevent its complete processing [40]. HSP70 has been implicated in the inhibition of apoptosome formation [20,41], but may also inhibit caspase-dependent events that occur later in apoptosis [42]. Chromosomal DNA is digested by caspase-activated-DNase/DNA fragmentation factor 
40 (CAD/DFF40) during the final stages of apoptosis, upon activation by caspase-3 [43]. The enzymatic activity and structural integrity of CAD/DFF40 was reported to be regulated by HSP70 and HSP40 [44]. Over-expression of these HSPs may prevent nuclear degradation regardless of up-stream pro-apoptotic events. We have recently reported in the same population of patients that RA-PBL sustain significant damage due to oxidative stress [32]. This may induce cellular stress responses which increase the expression of HSPs, which could possibly modulate apoptotic signal cascades. This may have contributed to the lack of lymphocyte DNA fragmentation observed in our patient cohort, despite early signs of apoptosis. Earlier studies by Szodoray et al (2003) examined nuclear condensation as a measure of apoptosis in circulating RA T-lymphocytes bearing typical apoptotic markers (CD95/ Fas, Bax, Bcl-2 and TNF receptor) [45]. These investigations showed decreased levels of nuclear condensation in T-lymphocytes and were thus interpreted to have decreased rates of CD95/Fas mediated apoptosis. In addition, lymphocytes positive for Bax protein also showed decreased apoptosis frequency. The study concluded that the reduced susceptibility to CD95-mediated apoptosis may contribute to the expansion of an activated $\mathrm{CD} 4^{+}$ lymphocyte sub-population and thus to the maintenance of peripheral autoreactive T-cell clones in RA [45].

Furthermore, the molecular features of apoptosis measured in RA-PBL did not translate to reduced numbers of circulating lymphocytes in our patient cohort. This was indicated by a lack of statistically significant correlation between absolute lymphocyte counts and total PBL apoptosis. This observation supports the notion that the apoptotic program may not be fully executed despite early molecular signs of apoptosis in RA-PBL. Albeck et al (2008) recently reported that although cells may exhibit molecular hallmarks of apoptosis, they may not be committed to fully executing the program and may recover from pro-apoptotic signals [46]. Although the mechanisms of apoptosis recovery are not fully understood, caspase inhibition via the X-linked-inhibitor-of-apoptosis-protein (XIAP) and proteosomal degradation of executioner caspases seem to play a role [47]. Interestingly, Rehm et al (2006) also described a state in which cells may exist with partial caspase-dependent degradation of their proteomes without outward manifestations of apoptotic features [48]. In RA, it is likely that although apoptosis is initiated in circulating lymphocytes, these cells may not be committed to executing the molecular program fully and cellular interactions at the synovium exacerbate their anti-apoptotic phenotype. In addition, chronically elevated lymphocyte counts may occur as a result of apoptosis induced compensatory proliferation. Recent studies have elucidated non-apoptotic functions of both initiator and executioner caspases. These are involved in generating growth stimulating and compensatory cell proliferation signals via alternate MAPK cascades [49]. Death receptors have also been implicated in non-cytotoxic responses which include regulation of cell proliferation, growth stimulation and production of pro-inflammatory chemokines. Evidence already indicates that engagement of death receptors in the rheumatoid synovium promotes cell proliferation instead of cell death [50].

Although not fully understood, similar mechanisms may operate and contribute to the maintenance of autoreactive lymphocyte clones in autoimmune diseases where apoptosis is elevated in peripheral circulation.

\section{Acknowledgements}

D. Moodley was supported by scholarships from the National Research Foundation Prestigious Equity grant and Andrew Mellon Foundation. We thank Sr. N. G. Mkhize and Dr N. Patel (Rheumatology Clinic - Inkosi Albert Luthuli Central Hospital, Durban) for their assistance during recruitment and clinical assessments of patients. We also thank Ms A. Phulukdaree for her assistance with western blot analysis.

\section{Author details}

'Discipline of Medical Biochemistry, Faculty of Health Sciences, University of KwaZulu-Natal, Private Bag 7, Congella, 4013, Durban, South Africa.

${ }^{2}$ Department of Rheumatology, Nelson R Mandela School of Medicine, University of KwaZulu-Natal, Private Bag 7, Congella, 4013, Durban, South Africa.

\section{Authors' contributions}

DM conceived the study, conducted all experimental laboratory experiments, analysed data and prepared the draft manuscript. GM participated in the design of the study and preparation of the final draft. AC participated in the design of the study, data analysis and preparation of the final draft. All authors read and approved the final manuscript.

\section{Competing interests}

The authors declare that they have no competing interests.

Received: 21 April 2011 Accepted: 3 November 2011

Published: 3 November 2011

\section{References}

1. Gay S, Kuchen S, Gay RE, Neidhart M: Cartilage destruction in rheumatoid arthritis. Ann Rheum Dis 2002, 61(Suppl 2):ii87.

2. Firestein GS: The immunopathogenesis of rheumatoid arthritis. Curr Opin Rheumatol 1991, 3:398-406.

3. Serhan CN, Savill J: Resolution of inflammation: the beginning programs the end. Nat Immunol 2005, 6:1191-1197.

4. Feig C, Peter ME: How apoptosis got the immune system in shape. Eur J Immunol 2007, 37(Suppl 1):S61-70.

5. Baier A, Meineckel I, Gay S, Pap T: Apoptosis in rheumatoid arthritis. Curr Opin Rheumatol 2003, 15:274-279.

6. Pap T, Muller-Ladner U, Gay RE, Gay S: Fibroblast biology. Role of synovial fibroblasts in the pathogenesis of rheumatoid arthritis. Arthritis Res 2000, 2:361-367.

7. Salmon $M$, Scheel-Toellner $D$, Huissoon AP, Pilling D, Shamsadeen $N$, Hyde H, D'Angeac AD, Bacon PA, Emery P, Akbar AN: Inhibition of T cell apoptosis in the rheumatoid synovium. J Clin Invest 1997, 99:439-446.

8. Zhang J, Bardos T, Mikecz K, Finnegan A, Glant TT: Impaired Fas signaling pathway is involved in defective T cell apoptosis in autoimmune murine arthritis. J Immunol 2001, 166:4981-4986.

9. Schirmer M, Vallejo AN, Weyand CM, Goronzy JJ: Resistance to apoptosis and elevated expression of $\mathrm{BCl}-2$ in clonally expanded CD4+CD28- T cells from rheumatoid arthritis patients. J Immunol 1998, 161:1018-1025.

10. Nanki T, Hayashida K, El-Gabalawy HS, Suson S, Shi K, Girschick HJ, Yavuz S, Lipsky PE: Stromal cell-derived factor-1-CXC chemokine receptor 4 
interactions play a central role in CD4+ T cell accumulation in rheumatoid arthritis synovium. J Immunol 2000, 165:6590-6598.

11. Yanaba K, Bouaziz JD, Matsushita T, Magro CM, St Clair EW, Tedder TF: Blymphocyte contributions to human autoimmune disease. Immunol Rev 2008, 223:284-299

12. Venkateshan SP, Sidhu S, Malhotra S, Pandhi P: Efficacy of biologicals in the treatment of rheumatoid arthritis. a meta-analysis. Pharmacology 2009, 83:1-9

13. Tolusso B, De Santis M, Bosello S, Gremese E, Gobessi S, Cuoghi I, Totaro MC, Bigotti G, Rumi C, Efremov DG, Ferraccioli G: Synovial B cells of rheumatoid arthritis express ZAP-70 which increases the survival and correlates with the inflammatory and autoimmune phenotype. Clin Immunol 2009, 131:98-108.

14. Lindhout $E$, van Eijk $M$, van Pel M, Lindeman J, Dinant HJ, de Groot C: Fibroblast-like synoviocytes from rheumatoid arthritis patients have intrinsic properties of follicular dendritic cells. J Immunol 1999. 162:5949-5956.

15. Hayashida K, Shimaoka Y, Ochi T, Lipsky PE: Rheumatoid arthritis synovial stromal cells inhibit apoptosis and up-regulate BCl-xL expression by $\mathrm{B}$ cells in a CD49/CD29-CD106-dependent mechanism. J Immunol 2000, 164:1110-1116

16. Reparon-Schuijt CC, van Esch WJ, van Kooten C, Rozier BC, Levarht EW, Breedveld FC, Verweij CL: Regulation of synovial B cell survival in rheumatoid arthritis by vascular cell adhesion molecule 1 (CD106) expressed on fibroblast-like synoviocytes. Arthritis Rheum 2000, 43:1115-1121.

17. Bijl M, Horst G, Limburg PC, Kallenberg CG: Fas expression on peripheral blood lymphocytes in systemic lupus erythematosus (SLE): relation to lymphocyte activation and disease activity. Lupus 2001, 10:866-872.

18. Stummvoll GH, Aringer M, Smolen JS, Koller M, Kiener HP, Steiner CW, Bohle B, Knobler R, Graninger WB: Derangement of apoptosis-related lymphocyte homeostasis in systemic sclerosis. Rheumatology (Oxford) 2000, 39:1341-1350.

19. Rajaiah $R$, Moudgil KD: Heat-shock proteins can promote as well as regulate autoimmunity. Autoimmun Rev 2009, 8:388-393.

20. Beere HM, Wolf BB, Cain K, Mosser DD, Mahboubi A, Kuwana T, Tailor P, Morimoto Rl, Cohen GM, Green DR: Heat-shock protein 70 inhibits apoptosis by preventing recruitment of procaspase- 9 to the Apaf-1 apoptosome. Nat Cell Biol 2000, 2:469-475.

21. Takayama S, Reed JC, Homma S: Heat-shock proteins as regulators of apoptosis. Oncogene 2003, 22:9041-9047.

22. Liuzzo G, Giubilato G, Pinnelli M: T cells and cytokines in atherogenesis. Lupus 2005, 14:732-735.

23. Arnett FC, Edworthy SM, Bloch DA, MCShane DJ, Fries JF, Cooper NS, Healey LA, Kaplan SR, Liang MH, Luthra HS, et al: The American Rheumatism Association 1987 revised criteria for the classification of rheumatoid arthritis. Arthritis Rheum 1988, 31:315-324.

24. Fadok VA, Bratton DL, Frasch SC, Warner ML, Henson PM: The role of phosphatidylserine in recognition of apoptotic cells by phagocytes. Cell Death Differ 1998, 5:551-562.

25. Sprent J, Kishimoto $\mathrm{H}$ : The thymus and central tolerance. Philos Trans $R$ Soc Lond B Biol Sci 2001, 356:609-616.

26. Hoyne GF, Dallman MJ, Lamb JR: T-cell regulation of peripheral tolerance and immunity: the potential role for Notch signalling. Immunology 2000 100:281-288

27. Gougeon ML, Piacentini M: New insights on the role of apoptosis and autophagy in HIV pathogenesis. Apoptosis 2009, 14:501-508.

28. Cacciapaglia F, Spadaccio C, Chello M, Gigante A, Coccia R, Afeltra A, Amoroso A: Apoptotic molecular mechanisms implicated in autoimmune diseases. Eur Rev Med Pharmacol Sci 2009, 13:23-40.

29. Rueda B, Fernandez-Gutierrez B, Balsa A, Pacual-Salcedo D, Lamas JR, Raya E, Gonzalez-Gay MA, Martin J: Investigation of CD69 as a new candidate gene for rheumatoid arthritis. Tissue Antigens 2008, 72:206-210.

30. Walsh GM, Williamson ML, Symon FA, Willars GB, Wardlaw AJ: Ligation of CD69 induces apoptosis and cell death in human eosinophils cultured with granulocyte-macrophage colony-stimulating factor. Blood 1996, 87:2815-2821.

31. Fernandez-Gutierrez B, Hernandez-Garcia C, Banares AA, Jover JA: Characterization and regulation of CD69 expression on rheumatoid arthritis synovial fluid T cells. J Rheumatol 1995, 22:413-420.
32. Moodley D, Mody G, Patel N, Chuturgoon AA: Mitochondrial depolarisation and oxidative stress in rheumatoid arthritis patients. Clin Biochem 2008.

33. Adrain C, Creagh EM, Martin SJ: Apoptosis-associated release of Smac/ DIABLO from mitochondria requires active caspases and is blocked by Bcl-2. Embo J 2001, 20:6627-6636

34. Bao Q, Shi Y: Apoptosome: a platform for the activation of initiator caspases. Cell Death Differ 2007, 14:56-65.

35. Siegel RM, Muppidi J, Roberts M, Porter M, Wu Z: Death receptor signaling and autoimmunity. Immunol Res 2003, 27:499-512.

36. Wallach D, Varfolomeev EE, Malinin NL, Goltsev YV, Kovalenko AV, Boldin MP: Tumor necrosis factor receptor and Fas signaling mechanisms. Annu Rev Immunol 1999, 17:331-367.

37. Pandey P, Farber R, Nakazawa A, Kumar S, Bharti A, Nalin C, Weichselbaum R, Kufe D, Kharbanda S: Hsp27 functions as a negative regulator of cytochrome c-dependent activation of procaspase-3. Oncogene 2000, 19:1975-1981.

38. Garrido C, Bruey JM, Fromentin A, Hammann A, Arrigo AP, Solary E: HSP27 inhibits cytochrome c-dependent activation of procaspase-9. Faseb $J$ 1999, 13:2061-2070

39. Bruey JM, Ducasse C, Bonniaud P, Ravagnan L, Susin SA, Diaz-Latoud C, Gurbuxani S, Arrigo AP, Kroemer G, Solary E, Garrido C: Hsp27 negatively regulates cell death by interacting with cytochrome c. Nat Cell Biol 2000, 2:645-652

40. Kamradt MC, Lu M, Werner ME, Kwan T, Chen F, Strohecker A, Oshita S, Wilkinson JC, Yu C, Oliver PG, et al: The small heat shock protein alpha Bcrystallin is a novel inhibitor of TRAlL-induced apoptosis that suppresses the activation of caspase-3. J Biol Chem 2005, 280:11059-11066.

41. Saleh A, Srinivasula SM, Balkir L, Robbins PD, Alnemri ES: Negative regulation of the Apaf-1 apoptosome by Hsp70. Nat Cell Biol 2000, 2:476-483

42. Jaattela M, Wissing D, Kokholm K, Kallunki T, Egeblad M: Hsp70 exerts its anti-apoptotic function downstream of caspase-3-like proteases. Embo 1998, 17:6124-6134

43. Elmore S: Apoptosis: a review of programmed cell death. Toxicol Pathol 2007, 35:495-516.

44. Sakahira H, Nagata S: Co-translational folding of caspase-activated DNase with $\mathrm{Hsp70}, \mathrm{Hsp} 40$, and inhibitor of caspase-activated DNase. J Biol Chem 2002, 277:3364-3370

45. Szodoray P, Jellestad S, Nakken B, Brun JG, Jonsson R: Programmed cell death in rheumatoid arthritis peripheral blood T-cell subpopulations determined by laser scanning cytometry. Lab Invest 2003, 83:1839-1848.

46. Albeck JG, Burke JM, Aldridge BB, Zhang M, Lauffenburger DA, Sorger PK: Quantitative analysis of pathways controlling extrinsic apoptosis in single cells. Mol Cell 2008, 30:11-25.

47. Albeck JG, Burke JM, Spencer SL, Lauffenburger DA, Sorger PK: Modeling a snap-action, variable-delay switch controlling extrinsic cell death. PLOS Biol 2008, 6:2831-2852

48. Rehm M, Huber HJ, Dussmann $\mathrm{H}$, Prehn JH: Systems analysis of effector caspase activation and its control by X-linked inhibitor of apoptosis protein. Embo J 2006, 25:4338-4349.

49. Yi CH, Yuan J: The Jekyll and Hyde functions of caspases. Dev Cell 2009, 16:21-34.

50. Morel J, Audo R, Hahne M, Combe B: Tumor necrosis factor-related apoptosis-inducing ligand (TRAIL) induces rheumatoid arthritis synovial fibroblast proliferation through mitogen-activated protein kinases and phosphatidylinositol 3-kinase/Akt. J Biol Chem 2005, 280:15709-15718.

doi:10.1186/1476-9255-8-30

Cite this article as: Moodley et al:: Initiation but no execution modulation of peripheral blood lymphocyte apoptosis in rheumatoid arthritis - a potential role for heat shock protein 70. Journal of Inflammation 2011 8:30. 